\title{
A short-term divergent selection for resistance to Teladorsagia circumcincta in Romanov sheep using natural or artificial challenge
}

\author{
Lucas Gruner $^{\mathrm{a} *}$, Jacques Bouix ${ }^{\mathrm{b}}$, Jacqueline Vu TiEn Khang ${ }^{\mathrm{b} * *}$,

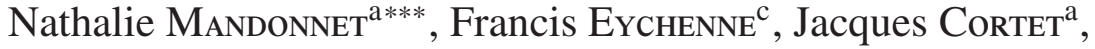 \\ Christine SAuvÉ ${ }^{\mathrm{a}}$, Claude Limouzin ${ }^{\mathrm{d}}$ \\ ${ }^{a}$ Bioagresseurs, santé et environnement, Institut national de la recherche agronomique, \\ 37380 Nouzilly, France \\ b Station d'amélioration génétique des animaux, Inra, BP 27, 31326 Castanet-Tolosan, France \\ ${ }^{c}$ Domaine de Langlade, Inra, Pompertuzat, 31450 Montgiscard, France \\ ${ }^{\mathrm{d}}$ UE Pathologie aviaire et parasitologie, Inra, 37380 Nouzilly, France
}

(Received 2 April 2003; accepted 29 October 2003)

\begin{abstract}
This experiment was conducted to assess the efficiency of selection on the basis of response to artificial challenges in order to breed sheep resistant to natural infection. A shortterm divergent selection process was designed to estimate the genetic parameters of these two traits. Two flocks, including 100 Romanov ram lambs each, were challenged in 1990 when they were 6 months old. One flock received three artificial infections with 20000 third-stage Teladorsagia circumcincta larvae, at intervals of 7 weeks. Faecal egg counts (FEC) were performed on Days 22, 25 and 28 post infection (p.i.) and the animals were drenched on Day 28 p.i. The other flock was grazed for 5 months on a pasture contaminated with the same species. Faecal samples were taken from the lambs at similar ages. About 5 rams with the lowest FEC and 5 with the highest FEC were selected in each flock and mated with unselected ewes. Their offspring (200 animals) were challenged in 1992, half in the same way as their sires, and the other half by the other method. Because of a drought in the summer of 1990, it was necessary to repeat part of the experiment, and in 1992 the 5 and 8 rams with the lowest and highest FEC, respectively, were selected from the offspring challenged on the pasture in 1992 and were mated with unselected ewes. Their progeny (about 80 animals) were challenged in 1994, half by natural infection, half by artificial infection. The mean FEC of the flock increased from the first to the third artificial infection. The natural infection was highly variable in different years, reflecting the difficulty of assessing resistance using this mode of challenge. Genetic parameters were estimated using animal models and REML solutions. The repeatabilities of the FEC following

*Corresponding author: gruner@tours.inra.fr

** Present address: 28 rue du Couvent, L-1363 Howald, Luxembourg

*** Present address: Inra, Unité de recherches zootechniques, 97170 Petit-Bourg, French West Indies
\end{abstract}


artificial and natural infection were 0.49 and 0.70 respectively within a period of one week, and 0.22 and 0.41 respectively for periods separated by intervals of 7 weeks; the heritabilities of the single egg count were 0.22 and 0.38 respectively. The genetic correlation was 0.87 : the FEC recorded under natural or artificial infection appear to depend on the same genetic potential.

host resistance / sheep-nematoda / Teladorsagia circumcincta / genetic parameters

\section{INTRODUCTION}

The development of strains of gastrointestinal trichostrongyles resistant to anthelmintics is a widespread phenomenon that is becoming prevalent in Europe. In France, cases of resistance to benzimidazole have been reported in about one sheep flock out of two, and in nearly all goat herds $[9,10]$. Consequently, alternative control measures are required to limit the use of anthelmintic treatments. Since genetic variability of resistance to gastrointestinal nematodes is known to exist $[14,19]$, selecting for resistant animals could provide an alternative to treatment with anthelmintics.

In many cases, the estimates of heritabilities have been derived from experiments in which sheep had been artificially challenged with single doses of infective larvae. The resistance expressed under field conditions is however of more interest to the sheep industry. The abilities of an animal to resist to a single oral dose of infective larvae or a continuous ingestion of larvae on the pasture are not necessarily controlled by the same set of genes. Moreover, genetic resistance to natural infection may be influenced by grazing behaviour, which could also have a genetic component.

The main purpose of this experiment was to test the effectiveness of selection on the basis of the response to artificial infections (which are easier to control and to standardise) by improving genetic resistance to natural infection (which is the objective of selective breeding in the field). The reason for this is because in France, there is a collective selection scheme for each breed with a central farm where 100 to 700 young males issued from elite parents in the participating flocks gather during 10 weeks. The best among them are the future rams for the organisation and the very best are the future elite sires used in artificial insemination. This very small number of animals has the totality of the genes of the future selected populations (from 5000 to 30000 ewes depending on the breed). So, an efficient and economical way for improving resistance would be a standardised fast test at the end of the 10 weeks stay in the central farm. Artificial challenges seem to be preferable to natural infection for assessing resistance in a breeding scheme, because the duration and impact of infection can be minimised. Furthermore, a timetable for measurements can be 
scheduled independently of the weather. Sheep selected on the basis of their response to artificial challenges have been shown to respond similarly when exposed to natural infection $[13,34,36]$, but no estimates of the genetic correlation have been made.

The trait considered in this work was the response to single-species infections with Teladorsagia circumcincta. Single-species challenges were preferred to mixed challenges in order to avoid the possible interactions between species. T. circumcincta is one of the most prevalent parasites in temperate climates, and progress has been made on the genetic resistance of this species, especially in the context of a single-species infection. The repeatabilities of the faecal egg count (FEC) have been estimated in artificial infections [30], but previously genetic parameters have only been estimated in natural mixed infections $[2,11,23,24,29,33]$, or predominantly $T$. circumcincta infection under Scottish conditions [6]. The criterion used for assessing resistance was the egg output, which is indirectly related to worm burden. It also has some inherent value as a breeding objective, since it reflects the level of contamination of the pasture, which in turn determines the level of exposure of a grazing flock to parasitic infection. Gruner et al. [19] have confirmed the value of FEC in predicting the infection risk of the flock.

We wish to apply genetics to improve the resistance of sheep against parasites under natural conditions of infection by using the results from standardised artificial infections. The practical objective was to obtain an effective criterion for the individual testing of young males, as was done in French selection schemes. To do this, it was necessary to estimate the repeatability and heritability of the FEC after artificial challenges and after natural infection, and the genetic correlation between these two traits. The experiment was designed to estimate these genetic parameters as accurately as possible within the limitations imposed by the scale of the experiment.

\section{MATERIALS AND METHODS}

\subsection{Experimental designs and resources considered}

Two possible designs were considered: one was based on the analysis of half-sib groups sired by rams chosen as representative of the variability of the experimental flock, the other on the "realised genetic parameters" following a short-term divergent selection. The major experimental limitation was the number of animals that could be measured annually: one hundred for each of these modes of infection. However, it was possible to assess twice as many 
animals by scheduling the experiment over two distinct years. Another limiting factor arose from the impossibility of measuring both traits in the same animal, because the first infection could obviously modify the response to the subsequent one.

The first design was based on $p$ families each of $2 n$ half-sib sires, $n$ measured for one trait and $n$ for the other, so that the total number of animals measured for each trait was $p . n=200$. Sampling variances of the heritability estimates are given by Robertson [25,26]; for genetic correlations the sampling variances were adapted from Tallis [32] for a situation in which two traits were measured in different animals (Appendix A).

In the second design, 200 males of the first generation (G1) were initially assessed: 100 for each trait. In each of these two subsets, the $n$ highest and $n$ lowest ranking individuals were selected. Each of these four groups was mated with unselected females in order to produce 50 lambs (G2) from which one elementary group of $m=25$ was artificially challenged and another of $m=25$ was naturally infected. As in the previous design, 400 animals were assessed (totalling 200 for each trait). The genetic parameters were estimated from direct and correlative responses to selection (Fig. 1). Sampling variances of estimates were derived from Hill [20,21], taking into account the distinctive features of the design considered here (Appendix B).

The standard errors of heritability and genetic correlation were computed for the two designs considered (with the optimal structure in each case), assuming that the two traits have the same heritability (Figs. 2 and 3). The design based on a one-generation divergent selection gave greater precision unless there was a very low heritability or a very high genetic correlation. The published estimates of heritabilities ranged from moderate to high (0.3 to 0.5), and so, a divergent selection procedure was chosen. The number of individuals, $N^{\prime}$, in the four groups of G1 sires selected was fixed at 5: this seemed to be close to the optimum number under the most likely assumptions regarding real values of the unknown parameters. Furthermore, a smaller number could involve a risk of selecting animals expressing extreme phenotypes because of non-genetic factors. Since the G1 males were randomly sired from 21 known $\mathrm{G} 0$ rams, the protocol realised was in fact a combination of the two designs.

\subsection{Experimental design and animals used}

Romanov sheep were used because of the high prolificacy of the ewes, and the high susceptibility of the breed towards gastrointestinal strongyles, compared with that of local breeds, such as the Lacaune [17] or Mérinos 


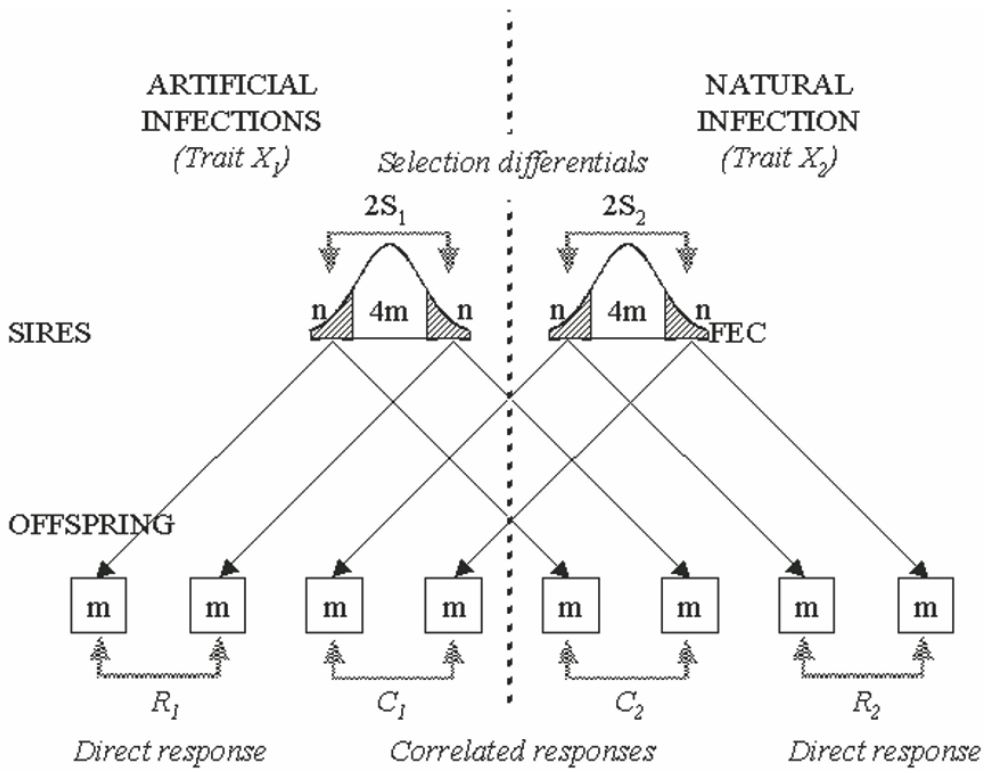

Figure 1. Diagram of a short-term divergent selection design. Selection is carried out on a single generation on the basis of two traits $\left(X_{1}=\right.$ resistance to artificial infections, $X_{2}=$ resistance to natural infection). The arrows indicate procreation of progeny groups by sires selected as resistant or susceptible for the trait $X_{1}$ or $X_{2}$. The selection criterion is the mean number of eggs per gram of faeces (epg). The "realised" genetic parameters are the following:

- heritabilities of the traits $X_{1}$ and $X_{2}: h_{1}^{2}=2 R_{1} / 2 S_{1}$ and $h_{2}^{2}=2 R_{2} / 2 S_{2}$

- genetic correlation between the traits $X_{1}$ and $X_{2}: \hat{r}=\sqrt{\frac{C_{2}}{R_{1}} \frac{C_{1}}{R_{2}}}$.

d'Arles [18]. A short-term divergent selection experiment was initiated in 1990 for the traits, resistance to natural infection and resistance to artificial challenges. To maximise the genetic variability of these two traits, 21 Romanov rams (generation G0) from different families were mated with 130 Romanov ewes (6-7 per ram) from the experimental flock of Langlade (Inra, SW of France). At 6 months of age, 97 and 95 ram lambs resulting from this mating (generation G1) and reared in penned conditions free of nematode parasites, were allocated to each of two flocks matched according to their sires and their weights (Fig. 4). In 1990, one of these flocks was given three artificial infections with $T$. circumcincta according to the protocol described below, and the other was grazed on a pasture contaminated with the same isolate of parasite. The resistance was measured using FEC and, at the end of the 5-month experimental period, about 5 rams with the lowest FEC values and 5 with the 


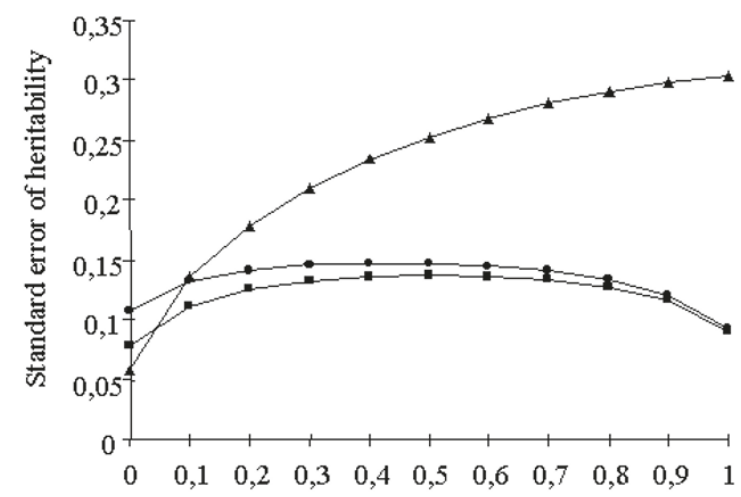

$\downarrow$ A : 200 animals in sire half-sib families

$\rightarrow$ B : divergent selection with $100 \mathrm{G} 1$ males and $100 \mathrm{G} 2$ sired from extreme G1

- $\mathrm{C}$ : as B but G1 males are structured in sire half-sib families

Figure 2. Standard error of the estimated heritability according to the real value of this parameter for the two designs considered (for a total of 200 animals measured for the trait under consideration).

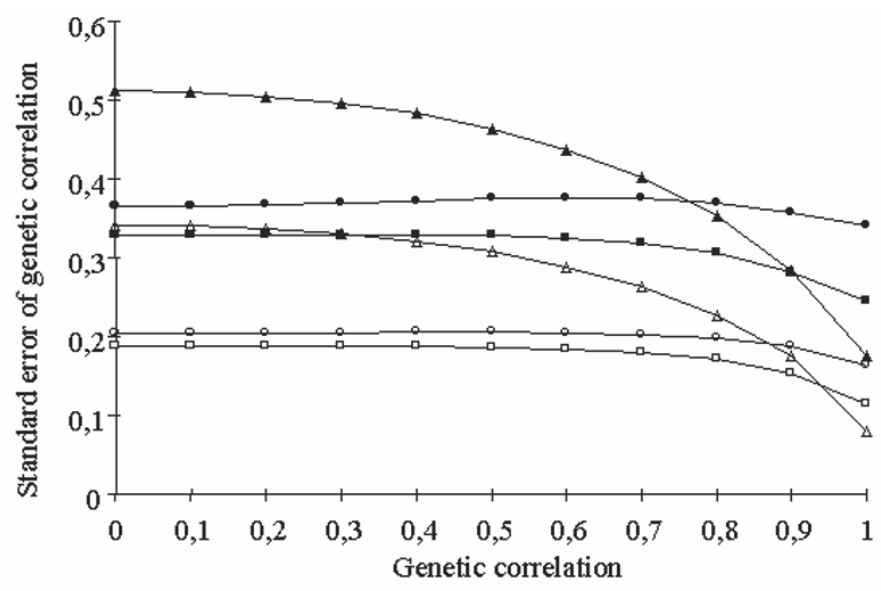

$\begin{array}{lll}\rightarrow \mathrm{A}: \text { Half-sib design }(\mathrm{h} 2=0.3) & \rightarrow \mathrm{A}: \text { Half-sib design }(\mathrm{h} 2=0.6) \\ \rightarrow \mathrm{B}: \text { Divergent selection }(\mathrm{h} 2=0.3) & \rightarrow \mathrm{B}: \text { Divergent selection }(\mathrm{h} 2=0.6) \\ \rightarrow \mathrm{C}: \mathrm{aA}+\mathrm{bB}(\mathrm{h} 2=0.3) & \rightarrow \mathrm{C}: \mathrm{aA}+\mathrm{bB}(\mathrm{h} 2=0.6)\end{array}$

Figure 3. Standard error of the estimated genetic correlation according to the real value of this parameter for the two designs considered (for a total of 200 animals measured for each trait), under several assumptions regarding the heritability $h^{2}$ of the two traits. 


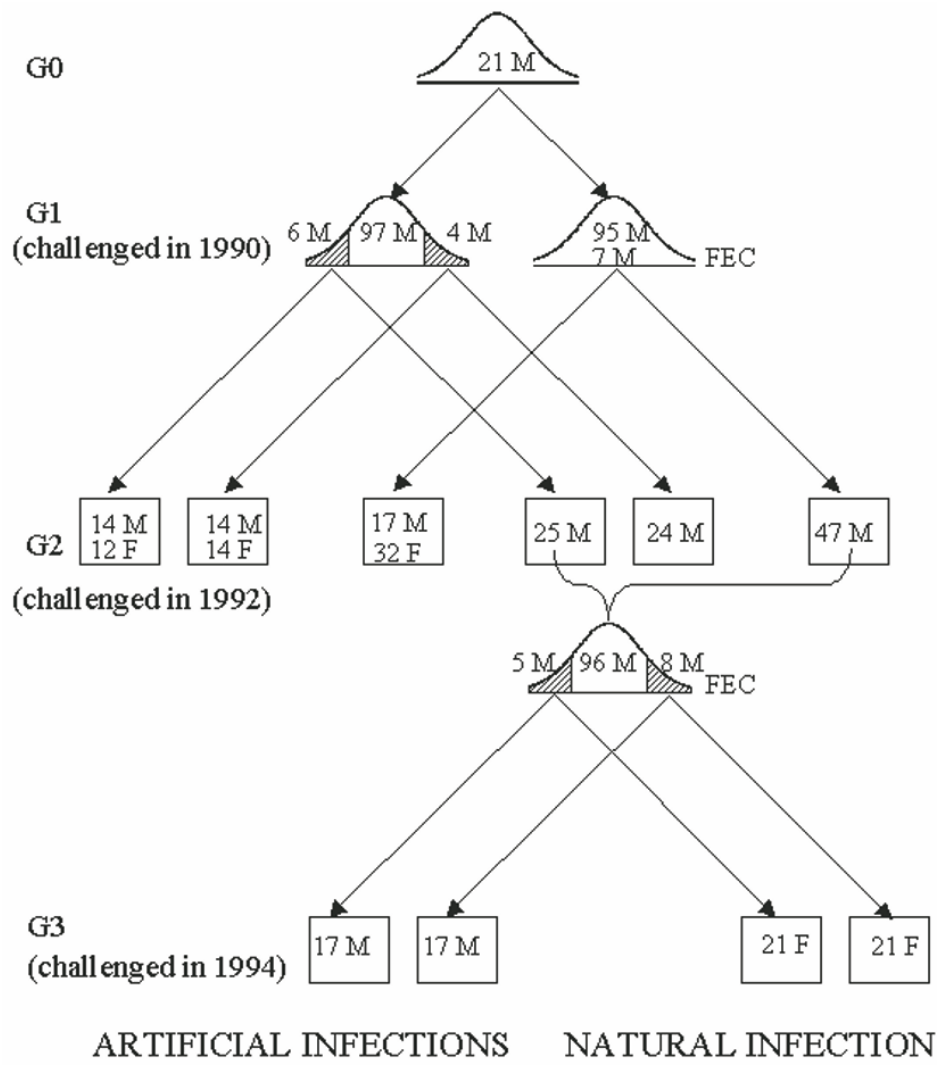

Figure 4. Realised experimental design with the number of animals $(\mathrm{M}=$ males, $\mathrm{F}=$ females) in each generation (G0 to G3). Arrows indicate the procreation of the progeny groups by sires selected as resistant or susceptible after artificial challenges or natural infection with $T$. circumcincta.

highest FEC values (classified as "resistant" and "susceptible" respectively) were selected from each flock. These four groups of rams were subsequently mated with unselected ewes, and produced about 50 lambs per group (generation G2). In 1992, half of the lambs from each group were challenged in the same way as their sires, and the other half was tested using the alternative challenge. Mating was intended to produce 200 ram lambs, however, this was not achieved. So we used 141 ram lambs plus 58 ewe lambs, which were added to a grazing flock comprising 96 ram lambs (it was not possible to manage two separate grazing flocks, one of each sex), and a housed flock comprising all the remaining lambs (45 rams and 58 ewe lambs) for artificial infection. Accidental mating occurred before the sexes were separated when the lambs were 
101 (80 to 139) days old, as a result of which 26 ewes became pregnant. The resultant lambs were separated from their mothers at birth.

The summer drought in 1990 limited the larval population on the pasture: the level of infection of the animals (mean and variance) was insufficient to allow us to assess their resistance. This part of the experiment was therefore repeated: the 5 most resistant and the 8 most susceptible rams from the G2 flock (tested using natural infection in 1992) were selected in April 1993 and mated with 80 unselected ewes. Each group of rams produced about 40 weaned lambs of each sex (generation G3). In 1994, the ram lambs were tested using artificial infection, and the ewe lambs were tested using natural infection.

\subsection{Artificial infection}

At 6 months of age, each lamb received a first dose of 20000 infective, thirdstage (L3) T. circumcincta larvae per os (from a field isolate obtained at Le Merle, in the South of France) and was treated 4 weeks later with fenbendazole (10 $\mathrm{mg} \cdot \mathrm{kg}^{-1}$ live weight) to eradicate the infection. Seven weeks after this first infection, each animal received a second dose (20000 L3), and was similarly drenched 4 weeks later. A third infection with the same dose was carried out 7 weeks after the second one. Faecal samples were collected on Days 22, 25 and 28 post infection (p.i.) and in addition on Day 19 after the third infection in 1992 (Fig. 5). A dose of 20000 L3 was used in order to ensure good antigen stimulation, without any pathological effects with this strain of T. circumcincta. The first infection was carried out when the lambs were around 6 months old to ensure that the animals could express their individual resistance potential. Stear and Murray [29] observed that the heritability of FEC following natural (predominantly $T$. circumcincta) infection was critically dependent upon the age of the lambs and was higher after 4-5 months.

\subsection{Natural infection}

In Nouzilly (Inra, Central France, with an average annual rainfall of $740 \mathrm{~mm}$ ), a 5 ha pasture, left ungrazed for a year, was sown with fescue and rye grass in the preceding autumn to remove any remaining population of gastrointestinal infective larvae. This pasture was seeded in July and August 1990 by two successive groups of lambs previously infected with 15000 L3 larvae of $T$. circumcincta (the isolate used for the artificial infections). When the G1generation flock reached 5.5 months old, they grazed this pasture for 4 months from mid July 1990. Because of a drought during this summer, irrigation was 
ARTIFICIAL WFCTIONS

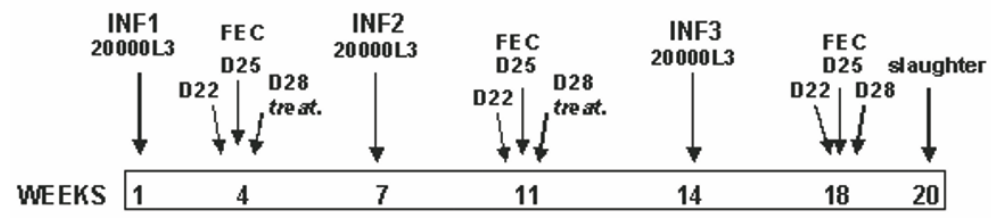

NATURAL INECTION

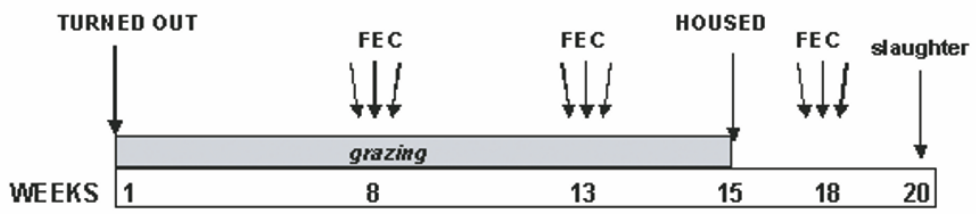

Figure 5. Protocol for assessing resistance to T. circumcincta in artificially and naturally infected flocks (INF $=$ artificial infection; treat. $=$ anthelmintic treatment $)$. The selection criterion is the mean of the six faecal egg counts measured during the second and third sampling periods.

required to maintain some grass production and larval development. Another rye grass pasture was used during the spring and summer of 1992 after it had been seeded with infected faeces (cultured for 14 days at $20^{\circ} \mathrm{C}$ ). When the G2 generation flock was 6 months old, it was put out to graze the pasture for 5 months from mid April 1992. The pasture used in 1990, and then not grazed in 1992 or 1993, was contaminated by groups of lambs previously infected with 15000 L3 of T. circumcincta grazing in October-November and in AprilMay 1994. When the G3 generation flock was 7 months old, it grazed this pasture for 5 months from mid April 1994. The animals received no anthelmintic treatment at any time during the experiment. Three sampling periods for FEC were chosen, so that both the artificially- and naturally-challenged flocks were sampled at the same age (Fig. 5).

To estimate the number of infective larvae ingested weekly by grazing animals, the pasture in each paddock was sampled on the first and last day of the grazing period and the larvae were extracted and counted [16]. At the same time, grass availability was estimated by collecting one square meter (in ten bands of $1 \mathrm{~m} \times 0.1 \mathrm{~m}$ ) and weighing it before and after drying for $24 \mathrm{~h}$ at $100{ }^{\circ} \mathrm{C}$. The amount of herbage ingested by the flock was calculated as the difference in the availability between the first and last day on the paddock, after correcting for pasture growth during the grazing period, estimated using 
six $1 / 4 \mathrm{~m}^{2}$ sheep-exclusion cages. This estimate gave acceptable data in 1990, but not in 1992, when the estimated feed availability was too high. The dead material in the pasture in 1992 was not taken into account, leading to an overestimation of the amount of grass ingested. Therefore, an estimated value of $1 \mathrm{~kg}$ dry matter per animal per day was used, and multiplied by the mean number of L3/kg dry matter to estimate the number of L3 larvae ingested each week during grazing. Similar estimates were obtained in 1994.

\subsection{Measurements}

\subsubsection{Faecal egg counts}

Individual egg counts were processed using McMaster slides with saturated magnesium sulphate as the flotation liquid. FEC were expressed as the number of eggs per gram of faeces (epg).

\subsubsection{Selection criterion}

As suggested by Woolaston et al. [37], the genetic ability to withstand a parasitic infection can only be expressed if the animal has previously been exposed to the parasite in order to "prime" its resistance mechanisms. The first artificial infection was therefore considered to act as a vaccination. Only the second and third infections were considered to be challenges: the animals were selected on the basis of the mean of the 6 FEC found following these infections. Similarly, in the naturally challenged flocks, the selection criterion was the mean of 6 FEC measured during the second and third sampling periods.

\subsection{Statistical analyses}

Data underwent preliminary analysis using the SAS/STAT package [27]. To normalise the FEC data, the Univariate procedure indicated that a square root transformation gave better results than a logarithmic transformation, so the counts were subjected to square root transformation. A standardisation of the variance for the four last measurements after artificial infection was performed, because there was greater variability in the years 1990 and 1992. Variance and correlation analyses were performed using the general linear model procedure. The effects of litter size and rearing rank (single, multiple or artificial) on FEC were examined, but they were not significant and therefore were ignored in 
Table I. Models of data analysis.

\begin{tabular}{|c|c|c|c|c|c|}
\hline \multirow[b]{2}{*}{ Model } & \multirow{2}{*}{$\begin{array}{l}\text { Information } \\
\text { analysed }\end{array}$} & \multicolumn{2}{|c|}{ No of data } & \multirow{2}{*}{$\begin{array}{c}\text { Traits analysed } \\
\text { by mode of infection }\end{array}$} & \multirow[t]{2}{*}{ Estimated parameters } \\
\hline & & Natural & Artific. & & \\
\hline 1 & FEC & 828 & 1404 & FEC 4 to 9 & $\sigma^{2} \mathbf{a}, \sigma^{2} \mathbf{i}, \sigma^{2} \mathbf{q}, \mathrm{r}_{\mathrm{g}}, \mathrm{r}_{\mathrm{w}}, \mathrm{r}_{\mathrm{b} 1}$ \\
\hline 2 & Infection & 276 & 468 & $\begin{array}{l}\text { Mean of FEC } 4 \text { to } 6 \text {, } \\
\text { Mean of FEC } 7 \text { to } 9 \text {, }\end{array}$ & $\sigma^{2} \mathbf{a}, \sigma^{2} \mathbf{i}, \mathrm{r}_{\mathrm{g}}, \mathrm{r}_{\mathrm{b} 2}$ \\
\hline 3 & Animal & 138 & 234 & Mean of FEC 4 to 9 & $\sigma^{2} \mathbf{a}, \mathrm{r}_{\mathrm{g}}$ \\
\hline
\end{tabular}

$\sigma^{2} \mathbf{a}$ : additive variance; $\sigma^{2} \mathbf{i}$ : individual permanent environment variance; $\sigma^{2} \mathbf{q}$ : worm population variance; $\mathrm{r}_{\mathrm{b} 1}$ : between period repeatability in model $1=\left(\sigma^{2} \mathbf{a}+\sigma^{2} \mathbf{i}\right) /\left(\sigma^{2} \mathbf{a}+\sigma^{2} \mathbf{i}+\sigma^{2} \mathbf{q}+\sigma^{2} \mathbf{e}_{1}\right)$; $\mathrm{r}_{\mathrm{w}}$ : within period repeatability in model $1=\left(\sigma^{2} \mathbf{a}+\sigma^{2} \mathbf{i}+\sigma^{2} \mathbf{q}\right) /\left(\sigma^{2} \mathbf{a}+\sigma^{2} \mathbf{i}+\sigma^{2} \mathbf{q}+\sigma^{2} \mathbf{e}_{1}\right)$; $\mathrm{r}_{\mathrm{b} 2}$ : between period repeatability in model $2=\left(\sigma^{2} \mathbf{a}+\sigma^{2} \mathbf{i}\right) /\left(\sigma^{2} \mathbf{a}+\sigma^{2} \mathbf{i}+\sigma^{2} \mathbf{e}_{2}\right) ; \mathrm{r}_{\mathrm{g}}$ : genetic correlation between the two traits.

subsequent analyses. The data concerning the G2 generation artificially challenged in 1992 were analysed by a factor combining sex and reproductive status (ram lambs, dry ewes or lambing ewes). In addition to the lamb's sex, the fixed date of measurement effects according to the type of data under analysis, was considered.

Due to the unpredictable drought in the summer of 1990, FEC and their variance were very low after natural infection and were therefore ignored. For this reason, the experiment actually performed (Fig. 4) was more complicated than the design initially planned (Fig. 1). The genetic parameters were derived from an individual animal model using the REML VCE4 package, 4.2.5 1998 version [15], which permits the use of all experimental data, including the pedigree information concerning the $200 \mathrm{G} 1$-generation males. This gave a further advantage to the design based on divergent selection (versus the half-sib design). Only FEC recorded during the second and third periods were considered for estimating genetic parameters.

Three models (Tab. I) were used for the data analysis:

Model 1 took the elementary values of FEC into consideration, with six data per animal:

$$
\mathbf{y}=\mathbf{X b}+\mathbf{Z a}+\mathbf{W}_{\mathbf{1}} \mathbf{i}+\mathbf{W}_{\mathbf{2}} \mathbf{q}+\mathbf{e}
$$

where $\mathbf{y}$ is the vector of observations, $\mathbf{b}$ is the vector of the fixed effects of sex and day of FEC, $\mathbf{a}$ is the vector of random additive genetic effects, $\mathbf{i}$ is the vector of random individual environmental permanent effects, $\mathbf{q}$ is the vector of random worm population effects specific to a particular challenge, $\mathbf{e}$ is the 
vector of random residuals, and $\mathbf{X}, \mathbf{Z}, \mathbf{W}_{\mathbf{1}}$ and $\mathbf{W}_{\mathbf{2}}$ are the incidence matrices connecting $\mathbf{y}$ to the effects of the model.

Model 2 took the mean values of the 3 FEC during each measurement period into consideration, with 2 data per animal:

$$
\mathbf{y}=\mathbf{X b}+\mathbf{Z a}+\mathbf{W}_{\mathbf{1}} \mathbf{i}+\mathbf{e}
$$

with the same effects as in model 1, except that $\mathbf{b}$ is the vector of the fixed effects of sex and FEC period, and the vector of mean values and incidence matrices are 3 times smaller.

Model 3 took the overall mean values of the 6 FEC into consideration, with 1 datum per animal:

$$
\mathbf{y}=\mathbf{X b}+\mathbf{Z a}+\mathbf{e}
$$

with the same effects as in model 1, except that $\mathbf{b}$ is the vector of the fixed effects of sex, and the vector of mean values and incidence matrices are 6 times smaller.

\section{RESULTS}

\subsection{Infection of the flocks after artificial challenge}

The artificially infected flocks displayed similar results in terms of egg production in the three experimental years (Fig. 6). Egg output peaked earlier and higher after subsequent infections, indicating that the prepatent period had been shortened. For instance, on Day 28 after the third infection in 1990, about $20 \%$ of the FEC were zero: in these animals, the established female worms had probably laid their eggs some days earlier and then stopped laying or had been expelled.

\subsection{Infection of the flocks after a natural challenge}

In 1990, the mean FEC were low, with $95 \%$ and $65 \%$ of the zero values at the first and second sampling periods, respectively. These findings reflect the summer drought: the lambs had ingested roughly 18000 L3 by the end of the grazing season (Fig. 7). In contrast, the summer of 1992 was rainy, and the lambs ingested 10 times more larvae than in 1990. At the first sampling period, after the progressive ingestion of 20000 infective larvae in 4-5 weeks, FEC were higher (400 to $600 \mathrm{epg}$, see Fig. 7) than in the flock that received a single dose of 20000 L3 larvae (less than 100 epg, see Fig. 6). During the 


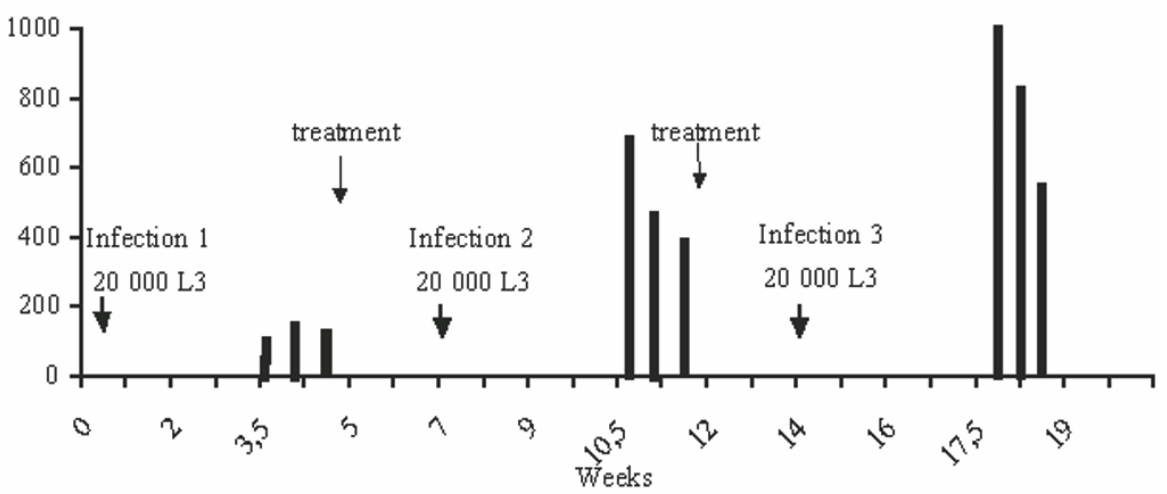

FEC Artificial infections in 1992
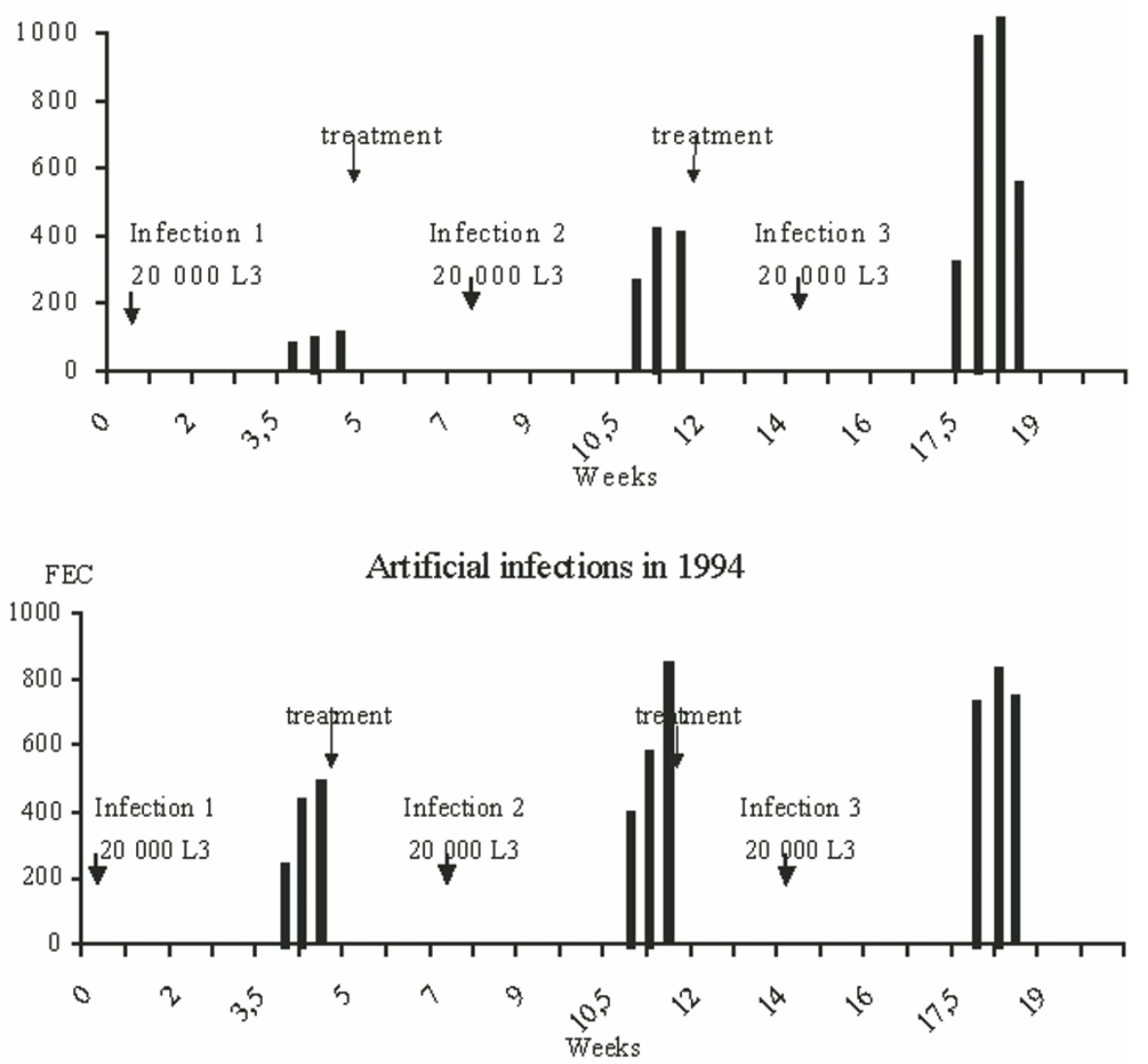

Figure 6. Mean (before adjustment for fixed effects) faecal egg counts (in eggs per g) in flocks artificially infected in 1990, 1992 and 1994 with three doses of 20000 third-stage larvae (L3) of T. circumcincta separated by a treatment (fenbendazole). 


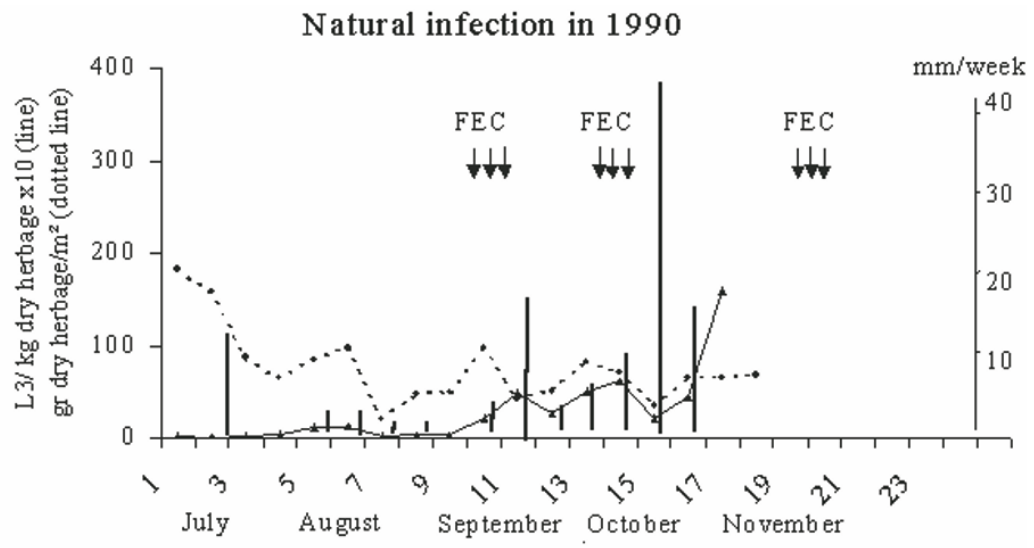

Natural infection in 1992

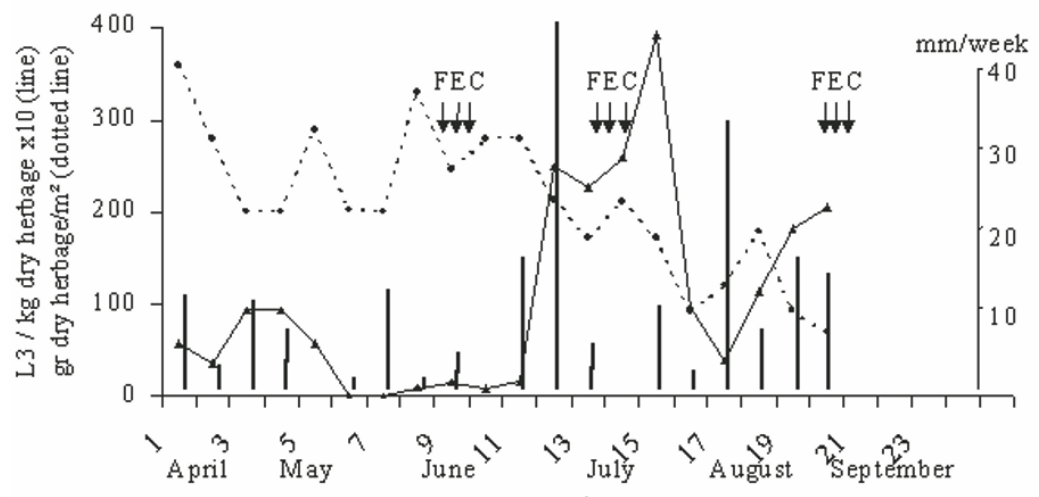

Natural infection in 1994

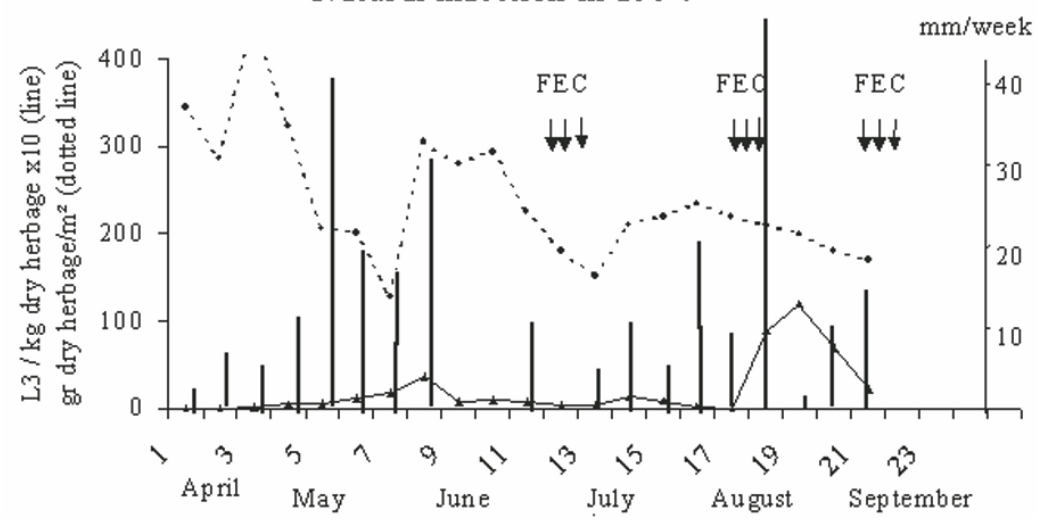

Figure 7. a - Weekly rainfall (bars), availability of grass (dotted line) and number of infective larvae (black line) on the pastures grazed by naturally infected flocks $(\mathrm{dm}=$ dry matter of grass). 

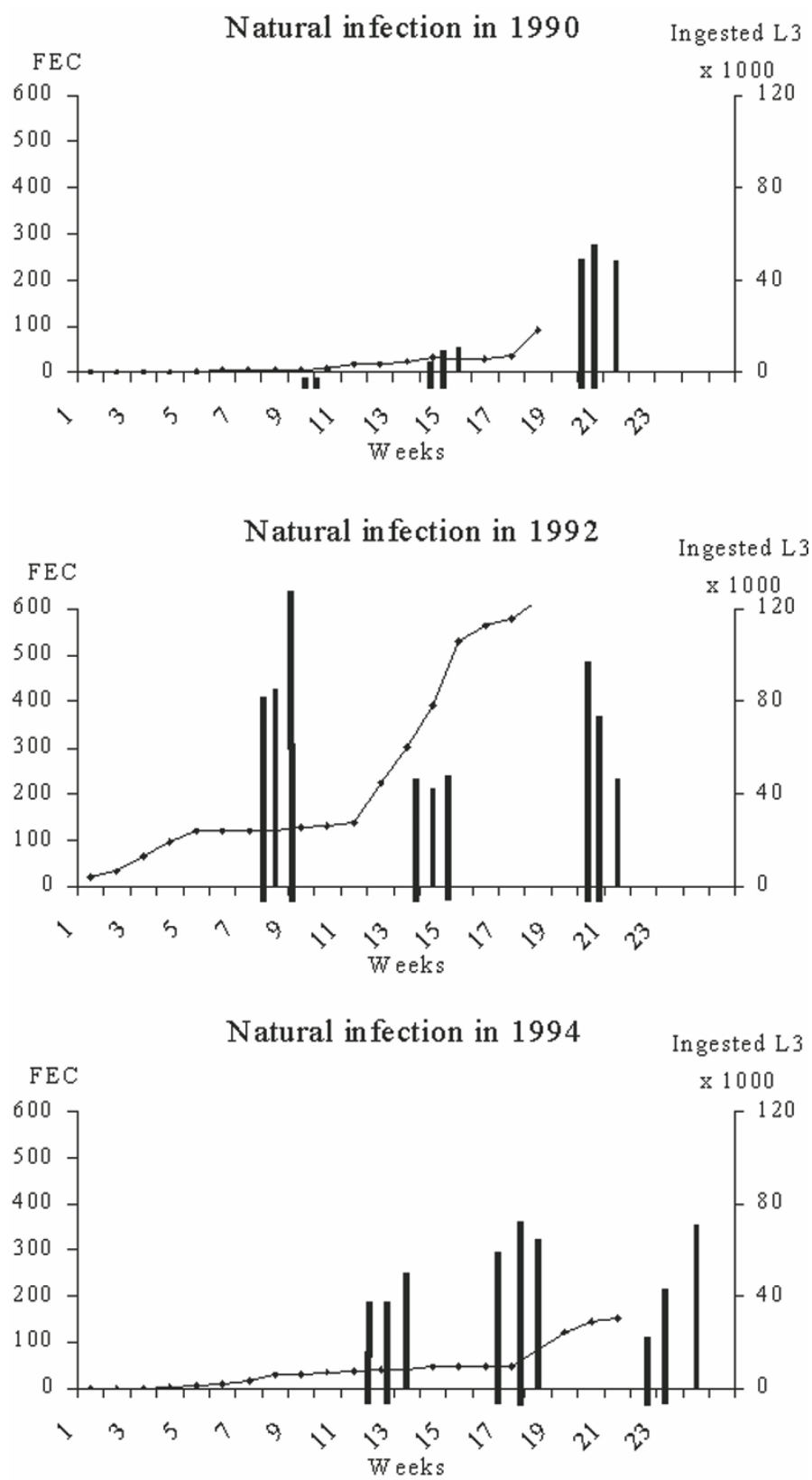

Figure 7. b - Accumulated mean numbers of ingested L3 larvae (black line) and mean faecal egg counts (bars) in flocks naturally infected during grazing in 1990, 1992 and 1994. 
third sampling period, FEC were relatively low in contrast to the very large numbers of incoming larvae. In 1994, pasture contamination was intermediate, with peaks in the spring and autumn.

\subsection{Effect of sex and the reproductive status of the ewes}

Egg output was significantly higher in the males than in the ewes at all three sampling periods (data not shown). The lambing time of the 26 ewe lambs accidentally mated when 4 months old corresponded to the first sampling period; FEC were significantly higher in the lambing ewes than in the dry ewes during that period, lower during the second period and similar during the third period.

\subsection{Genetic parameters}

The estimates of the variance components, and the genetic and phenotypic parameters for the different models are shown in Table II. The repeatability of the FEC was significantly higher within than between the sampling periods. Significantly higher values were also obtained for repeatabilities for natural than for artificial infections. The heritabilities and genetic correlation estimated by a REML on an animal model were generally consistent with the "parameters realised" (not shown here) estimated from the subsets of experimental data corresponding to a divergent selection. Two results were particularly striking. First, in artificial challenges, the heritability of the single egg count was equal to its repeatability between sampling periods $(0.22)$, but very similar values were also found after natural infection $\left(\mathrm{h}^{2}=0.38, \mathrm{r}=0.41\right)$. Second, there was a close genetic correlation between the FEC measured in the two modes of infection (0.87), and this was consistent across the three different models.

\section{DISCUSSION}

In artificially-infected flocks, egg output peaked earlier and rose more from the first to the third challenge. These changes in the pattern of egg excretion were probably due to the fenbendazole treatment. Benitez-Usher et al. [5] and Luffau et al. [22] have shown that thiabendazole treatment prevents the expression of immunity acquired by previous contact with Haemonchus contortus. Cabaj et al. [8] also found changes in the immune system of sheep that had been drenched with fenbendazole.

Two kinds of problems arose in the naturally-infected flocks: how to prepare and maintain a pasture infected with only one species (some undesired 
Table II. Estimated genetic parameters for faecal egg counts measured after artificial challenges or natural infection.

\begin{tabular}{cllllllllllll}
\hline & Type of & Information & \multicolumn{4}{c}{ Variance components } & \multicolumn{4}{c}{ Genetic parameters } \\
Model infection & analysed & $\sigma^{2} \mathbf{a}$ & $\sigma^{2} \mathbf{i}$ & $\sigma^{2} \mathbf{q}$ & $\sigma^{2} \mathbf{e}$ & $\sigma^{2} \mathbf{p}$ & $\mathrm{h}^{2}$ & $\mathrm{r}_{\mathrm{g}}$ & $\mathrm{r}_{\mathrm{b}}$ & $\mathrm{r}_{\mathrm{w}}$ \\
\hline 1 & Natural & FEC & 19.63 & 1.63 & 15.18 & 15.31 & 51.76 & $0.38 \pm 0.09$ & 0.87 & 0.41 & 0.70 \\
& Artificial & FEC & 10.90 & 0 & 13.48 & 24.99 & 49.37 & $0.22 \pm 0.03$ & \pm 014 & 0.22 & 0.49 \\
\hline 2 & Natural & infection & 19.63 & 1.64 & & 20.29 & 41.56 & $0.47 \pm 0.10$ & 0.87 & 0.51 \\
& Artificial & infection & 10.90 & 0 & & 21.81 & 32.71 & $0.33 \pm 0.04$ & \pm 0.12 & 0.33 & \\
\hline 3 & Natural animal & 19.80 & & 11.69 & 31.49 & $0.63 \pm 0.13$ & 0.86 & \\
& Artificial animal & 11.69 & & 10.02 & 21.71 & $0.54 \pm 0.09$ & \pm 0.13 & \\
\hline
\end{tabular}

$\sigma^{2} \mathbf{a}$ : additive variance; $\sigma^{2} \mathbf{i}$ : individual permanent environmental variance; $\sigma^{2} \mathbf{q}$ : worm population variance; $\sigma^{2} \mathbf{e}$ : residual variance; $\sigma^{2} \mathbf{p}$ : phenotypic variance; $\mathrm{r}_{\mathrm{g}}$ : genetic correlation between the two traits; $\mathrm{r}_{\mathrm{b}}$ : between period repeatability in model $1=\left(\sigma^{2} \mathbf{a}+\sigma^{2} \mathbf{i}\right) / \sigma^{2} \mathbf{p} ; \mathrm{r}_{\mathrm{w}}$ : within period repeatability in model $1=\left(\sigma^{2} \mathbf{a}+\sigma^{2} \mathbf{i}+\sigma^{2} \mathbf{q}\right) / \sigma^{2} \mathbf{p}$.

but minor infection with Cooperia oncophora occurred in 1992) and the variability of the larval population depending on the weather. These difficulties reinforced our view that it is important to compare the two methods of infection when assessing genetic resistance. FEC recorded in the summer of 1992 clearly demonstrated the influence of the mode of ingestion of infective larvae: the progressive ingestion of 20000 larvae on the pasture resulted in a higher egg output than the same quantity of larvae inoculated as a single dose. The relatively moderate egg production measured on the pasture during the third sampling period in the summer of 1992 after a very large quantity of infective larvae had been ingested, indicates the existence of a strong regulation of the worm population.

The significant effects of sex and reproductive status were consistent with the usual results reported in the literature: the higher resistance of females than males, and a periparturient rise in egg output [3].

The repeatability corresponds to the correlation between several measures performed on the same animal. This parameter is defined as the proportion of the variance of a single measurement in a flock that is attributable to permanent differences between individuals. These differences may be due to both genetic and individual permanent environmental factors, the difference between the values of repeatability and heritability expressing the non-genetic individual effects. Repeatability provides an assessment of the stability of the measurement, but also information about the usefulness of repeated determinations as discussed below in this section. As expected, the repeatability of 
the FEC was higher within than between periods ( 0.49 versus 0.22 for artificial infection; 0.70 versus 0.41 for natural infection). The FEC measured at intervals of several days in the same animal reflected very similar worm burdens. The within-period repeatability was higher in naturally- than in artificiallychallenged animals ( 0.70 versus 0.49$)$, indicating that the worm population was more stable in animals continuously exposed to natural infection on the pasture than in animals receiving a single dose of larvae. Similarly, the between-period repeatability was higher in naturally- than in artificially-challenged animals (0.41 versus 0.22 ), essentially because of the heritability values, and partly due to the persistence of a residual infection from one period to another in naturally-infected lambs. These animals continuously ingested larvae and were left undrenched for 5 months, so that the worm population built up progressively. In contrast, the use of an anthelmintic treatment between two successive challenges removed the worm burden from artificially-infected lambs.

In 9-month old sheep exposed to two successive artificial infections with 50000 infective $T$. circumcincta larvae separated by an anthelmintic treatment, Stear et al. [30] reported a within-infection FEC repeatability of 0.75 at 2-3 day intervals and of 0.50 at one week intervals, whereas the betweeninfection repeatability was 0.30 . Following an artificial challenge with 11000 H. contortus larvae, Woolaston et al. [37] reported a repeatability of 0.60 after an interval of one week. In wethers infected with $6000 \mathrm{H}$. contortus larvae, drenched and exposed to prolonged infection (1500 larvae per week for 13 weeks), Barger and Dash [4] reported a repeatability between single and extended infection(s) of 0.3 [4]. These estimates are close to those obtained in the present study, although in most cases they are higher.

In natural mixed infections (with the most prevalent genera Teladorsagia and Trichostrongylus), Cummins et al. [11] reported a mean repeatability of about 0.3 at 3-4 week intervals, whereas Baker et al. [2] estimated repeatabilities of 0.4 to 0.5 for FEC recorded at roughly 2 -month intervals. The repeatability estimated in the present study for natural single-species infection at 7-week intervals (0.41) was similar in magnitude to the estimate obtained by Baker et al. [2].

The estimates obtained for the heritabilities and genetic correlation were consistent with the assumptions on which the preliminary simulations were based. In the same way, the theoretical values of the sampling standard errors of genetic parameters were not so far off the corresponding real values in model 3, taking into account an underevaluation by VCE, of about $1 / \sqrt{2}$ observed by some users of the software. These estimates provide a post-factum justification of the choice of the experimental design. 
Although differences between breeds in their resistance to T. circumcincta have been known to exist for a long time, following either natural infection [31] or artificial challenge [28], no estimates of the heritability of FEC following single-species infection have been made. Heritabilities have been estimated only in natural mixed infections. Following natural infections involving mainly T. circumcincta, Piper [24], Cummins et al. [11], Stear and Murray [29] and Bishop et al. [6] reported heritability estimates of about $0.3,0.2$ and 0.2 to 0.4 respectively for a single count. Following infection in the field with Teladorsagia and Trichostrongylus as the most prevalent genera, the heritability of a single measurement of FEC was estimated at 0.34 by Watson et al. [33], 0.33 to 0.39 by Baker et al. [2] and 0.14 to 0.34 by Morris et al. [23]. In a situation where $T$. circumcincta and $H$. contortus are the prevalent species, Bouix et al. [7] found 0.33 and 0.25 for a single FEC at the end of the grazing season for the lambs and their mothers respectively. The heritability estimated in the present study under natural single-species infection (0.38) is consistent with the estimates previously obtained under natural mixed infections.

The heritability estimate for single FEC following artificial challenge with T. circumcincta (0.22) is similar in magnitude to those reported in sheep artificially challenged with $H$. contortus, which appear to be around 0.2 to $0.3[1,24,37]$. However, the present estimate seems to be lower than the heritabilities estimated for a single FEC following artificial challenge with $T$. colubriformis, which range from 0.3 to $0.6[12,35,37]$.

These heritabilities of a single FEC and its repeatability determine the value of repeated measurements: if a trait has a heritability $h^{2}$ and a repeatability $r$, adding a second measurement corresponds to a new trait with a new heritability equal to:

$$
\mathrm{h}^{2} \frac{\mathrm{n}}{1+(\mathrm{n}-1) \mathrm{r}} \text {. }
$$

So, with a very high repeatability close to 1 , adding new information does not at all increase the precision of the estimate because $1+(n-1) r=n$. For a given trait, the closer the values of $h^{2}$ and $r$ are (knowing that $r \geq h^{2}$ ), the greater is the increase of precision with a new measurement. Moreover, if $r \approx h^{2}$ the maximum of increase in precision is obtained when $h^{2}$ is weak, because $r$ is also weak.

Returning to our results, adding new information is more profitable in a strategy of artificial infection because of weaker values of each parameter: $\mathrm{h}^{2}$ goes from 0.22 to 0.54 (in model 3 , with two infections $\times 3 \mathrm{FEC}$ ) versus 0.38 to 0.63 in natural infection; this leads to final results that are not so different. The gain in precision is low when adding measurements after a given infection: 0.22 to 0.33 (in model 2 , with 1 infection $\times 3$ FEC) and 0.38 to 0.47 , 
respectively in artificial and natural strategies because of high repeatability within the infection ( 0.70 and 0.49$)$. In a more general way, the estimate parameters in model 1 are relevant for an optimisation of protocols for the evaluation of the genetic or individual resistance to parasites.

The most unexpected finding of this study was the estimation of the genetic correlation between egg outputs following either artificial or natural infection (0.87). Previous work also supports a positive correlation between responses to artificial and natural challenges, however, few estimates of the correlation have been previously published. Woolaston and Eady [35] have reported a genetic correlation of 0.72 between indoor pen-tested and paddock-tested animals (infected with T. colubriformis). Woolaston et al. [36] have reported differences in egg output observed between two lines of Merino sheep selected for increased or decreased resistance following artificial challenge with $H$. contortus larvae and an unselected line persisted after grazing naturally infected pastures (almost exclusively with $H$. contortus). Two lines of Perendale sheep selected for increased or reduced susceptibility to an artificial challenge with $H$. contortus responded similarly to natural mixed infection, with Haemonchus and Trichostrongylus as the dominant genera [34]. When lambs sired either by a genetically resistant ram or by susceptible rams (determined on the basis of the response of previous progeny to $H$. contortus artificial infection) were grazed on a pasture contaminated with three important species (H. contortus, T. colubriformis and $T$. circumcincta), the animals with the resistant genotype also displayed increased resistance to the natural mixed infection [13]. The present estimate supports the view that resistance assessed using artificial challenges and resistance measured after natural infection are largely determined by the same genes. This is of interest from a theoretical point of view, because it indicates that parasitological studies on the mechanisms of host resistance could be done using a very simple and standardised method (artificial infection) for animal screening. These findings are also of interest for breeders: they clearly demonstrate that breeding sheep for their resistance to natural infection could be done on the basis of the responses of the rams to artificial challenges, in circumstances where this offers logistical advantages.

\section{ACKNOWLEDGEMENTS}

We would like to thank the personnel of the Inra Domaine de Langlade (especially C. Baronchelli and G. Caste) where the sheep were bred and artificially infected flocks were maintained, and the personnel of Inra Nouzilly, where naturally infected flocks were kept (J. Auger, J. Besnard, T. Chaumeil 
and J. Méry). We are grateful to R.L. Baker for comments on the manuscript. This project was supported by the Inra AIP "Alternative aux antiparasitaires" and "Gènes de résistance aux maladies animales".

\section{REFERENCES}

[1] Albers G.A.A., Gray G.D., Piper L.R., Barker J.S.F., Le Jambre L.F., Barger I.A., The genetics of resistance and resilience to Haemonchus contortus infection in young Merino sheep, Int. J. Parasitol. 17 (1987) 1355-1363.

[2] Baker R.L., Watson T.G., Bisset S.A., Vlassof A., Douch P.G.C., Breeding sheep in New Zealand for resistance to internal parasites: research results and commercial application, in: Gray G.D., Woolaston R.R. (Eds.), Breeding for Disease Resistance in Sheep, Wool Research and Development Corporation, Parkville, Victoria, Australia, 1991, pp. 19-32.

[3] Barger I.A., Influence of sex and reproductive status on susceptibility of ruminants to nematode parasitism, Int. J. Parasitol. 23 (1993) 463-470.

[4] Barger I.A., Dash K.M., Repeatability of ovine faecal egg counts and blood packed cell volumes in Haemonchus contortus infections, Int. J. Parasitol. 17 (1987) 977-980.

[5] Benitez-Usher C., Armour J., Duncan J.L., Urquhart G.M., Gettinby G., A study of some factors influencing the immunization of sheep against Haemonchus contortus using attenuated larvae, Vet. Parasitol. 3 (1977) 327-342.

[6] Bishop S.C., Bairden K., Mc Kellar Q.A., Stear M.J., Genetic parameters for faecal egg count following, mixed, natural predominantly Ostertagia circumcincta infection and relationships with live weight in young lambs, Anim. Sci. 63 (1996) 423-428.

[7] Bouix J., Krupinski J., Rzepecki R., Nowosad B., Skrzyzala I., Roborzynski M., Fudalewicz-Niemczyk W., Skalska M., Malczweski A., Gruner L., Genetic resistance to gastrointestinal nematode parasites in Polish long-wool sheep, Int. J. Parasitol. 28 (1998) 1797-1804.

[8] Cabaj W., Stankiewicz M., Jonas W.E., Moore L.G., Fenbendazole and its effect on the immune system of the sheep, N.Z. Vet. J. 42 (1994) 216-220.

[9] Chartier C., Pors I., Hubert J., Rocheteau D., Benoit C., Bernard N., Prevalence of anthelmintic resistant nematodes in sheep and goats in western France, Small Rumin. Res. 29 (1998) 33-41.

[10] Chartier C., Soubirac F., Pors I., Silvestre A., Hubert J., Couquet C., Cabaret J., Prevalence of anthelmintic resistance in gastro-intestinal nematodes of dairy goats under extensive management conditions in southwestern France, J. Helminthol. 75 (2001) 325-330.

[11] Cummins L.J., Thompson R.L., Yong W.K., Riffkin G.G., Goddard M.E., Callinan A.P.L., Saunders M.J., Genetics of Ostertagia selection lines, in: Gray G.D., Woolaston R.R. (Eds.), Breeding for Disease Resistance in Sheep, Wool Research and Development Corporation, Parkville, Victoria, Australia, 1991, pp. 11-18. 
[12] Eady S.J., Woolaston R.R., Mortimer S.I., Internal parasite resistance of Merino flocks selected for production, Wool Technol. Sheep Breed. 42 (1994) 237-342.

[13] Gray G.D., Barger I.A., Le Jambre L.F., Douch P.G.C., Parasitological and immunological resistant Merino sheep on pastures contaminated with parasitic nematodes, Int. J. Parasitol. 22 (1992) 417-425.

[14] Gray G.D., Woolaston R.R., Eaton B.T., Breeding for Resistance to Infectious Diseases in Small Ruminants, Aus. Centre Int. Agric. Res., Canberra, Australia (1995).

[15] Groeneveld E., REML VCE - a multivariate model restricted maximum likelihood (co)variance component estimation package, in: Groeneveld E. (Ed.), Proceedings of an EC Symposium on Application of Mixed Linear Models in the Prediction of Genetic Merit in Pigs, Mariensee, Germany, 1993, pp. 83-102.

[16] Gruner L., Raynaud J.P., Technique allégée de prélèvements d'herbe et de numération pour juger de l'infestation des pâturages de bovins par les larves de nématodes parasites, Rev. Méd. Vét. 131 (1980) 521-529.

[17] Gruner L., Cabaret J., Sauvé C., Pailhories R., Comparative susceptibility of Romanov and Lacaune sheep gastrointestinal nematodes and small lungworms, Vet. Parasitol. 19 (1986) 85-93.

[18] Gruner L., Bouix J., Cabaret J., Boulard C., Cortet J., Sauvé C., Molénat G., Calamel M., Effect of genetic type, lactation and management on helminth infection of ewes in an intensive grazing system on irrigated pasture, Int. J. Parasitol. 22 (1992) 919-925.

[19] Gruner L., Cortet J., Sauvé C., Limouzin C., Brunel J.C., Evolution of nematode community in grazing sheep selected for resistance and susceptibility to Teladorsagia circumcincta and Trichostrongylus colubriformis: a 4-year experiment, Vet. Parasitol. 109 (2002) 277-291.

[20] Hill W.G., Design and efficiency of selection experiments for estimating genetic parameters, Biometrics 27 (1971) 293-311.

[21] Hill W.G., Estimation of realised heritabilities from selection experiments. I. Divergent selection, Biometrics 28 (1972) 747-765.

[22] Luffau G., Vu Tien Khang J., Bouix J., Nguyen T.C., Cullen P., Ricordeau G., Resistance to experimental infections with Haemonchus contortus in Romanov sheep, Genet. Sel. Evol. 22 (1990) 205-229.

[23] Morris C.A., Watson T.G., Bisset S.A., Vlassof A., Douch P.G.C., Breeding sheep in New Zealand for resistance or resilience to nematode parasites, in: Gray G.D., Woolaston R.R., Eaton B.T. (Eds.), Breeding for Resistance to Infectious Diseases of Small Ruminants, Aus. Centre Int. Agric. Res., Canberra, Australia, 1995, pp. 77-98.

[24] Piper L.R., Genetic variation in resistance to internal parasites, in: Merino Improvement Programs in Australia, Proceedings of a National Symposium, Leura, Aus. Wool Corp., Melbourne, Australia, 1987, pp. 351-362.

[25] Robertson A., Experimental design in the evaluation of genetic parameters, Biometrics 15 (1959) 219-226.

[26] Robertson A., The sampling variance of the genetic correlation coefficient, Biometrics 15 (1959) 469-485. 
[27] SAS ${ }^{\circledR}$ Institute Inc., SAS/STAT User's Guide, version 6, Fourth Edition, Cary, NC, USA (1989).

[28] Scrivner L.H., Breed resistance to ostertagiasis in sheep, J. Am. Vet. Med. Assoc. 144 (1964) 883-887.

[29] Stear M.J., Murray M., Genetic resistance to parasitic disease: particularly of resistance in ruminants to gastrointestinal nematodes, Vet. Parasitol. 54 (1994) 161-176.

[30] Stear M.J., Bishop S.C., Duncan J.L., McKellar Q.A., Murray M., The repeatability of faecal egg counts, peripheral eosinophil counts, and plasma pepsinogen concentrations during deliberate infections with Ostertagia circumcincta, Int. J. Parasitol. 25 (1995) 375-380.

[31] Stewart M.A., Miller R.F., Douglas J.R., Resistance of sheep of different breeds to infestation by Ostertagia circumcincta, J. Agric. Res. 55 (1937) 923-930.

[32] Tallis G.M., Sampling errors of genetic correlation coefficients calculated from analyses of variance and covariance, Aus. J. Stat. 1 (1959) 35-43.

[33] Watson T.G., Baker R.L., Harvey T.G., Genetic variation in resistance or tolerance to internal parasites in strains of sheep at Rotomahana, Proc. N.Z. Soc. Anim. Prod. 46 (1986) 23-26.

[34] Watson T.G., Hosking B.C., Hurford A.P., Mather B.C., Developments in breeding Perendale sheep for resistance or susceptibility to internal nematode parasites, Proc. N.Z. Soc. Anim. Prod. 52 (1992) 61-64.

[35] Woolaston R.R., Eady S.J., Australian research on genetic resistance to nematode parasites, in: Gray G.D., Woolaston R.R., Eaton B.T. (Eds.), Breeding for Resistance to Infectious Diseases of Small Ruminants, Aus. Centre Int. Agric. Res., Canberra, Australia, 1995, pp. 53-75.

[36] Woolaston R.R., Barger I.A., Piper L.R., Response to helminth infection of sheep selected for resistance to Haemonchus contortus, Int. J. Parasitol. 20 (1990) 1015-1018.

[37] Woolaston R.R., Windon R.G., Gray G.D., Genetic variation in resistance to internal parasites in Armidale experimental flocks, in: Gray G.D., Woolaston R.R. (Eds.), Breeding for Disease Resistance in Sheep, Aus. Wool Corp., Melbourne, Australia, 1991, pp. 1-9.

\section{APPENDIX A: ESTIMATION OF GENETIC PARAMETERS BY CORRELATION BETWEEN HALF-SIBS WITH TWO TRAITS MEASURED ON TWO DIFFERENT GROUPS OF ANIMALS}

\section{Experimental design}

Two traits were measured in two groups of $N=n . p$ paternal half-sibs, issued from $p$ sires, each with offspring of $n$ individuals. 


\section{Estimated parameters}

For the trait $X_{1}$, the sire component of additive variance is $\sigma_{a_{1}}^{2}$, and the within-sire residual variance is $\sigma_{e_{1}}^{2}$, with the heritability $\hat{h}_{1}^{2}=4 \frac{\sigma_{a_{1}}^{2}}{\sigma_{a_{1}}^{2}+\sigma_{e_{1}}^{2}}$, and the coefficient $\lambda_{1}=\frac{\sigma_{e_{1}}^{2}}{\sigma_{a_{1}}^{2}}$.

The components of covariance between the two traits are respectively $\operatorname{cov}\left(a_{1}, a_{2}\right)$ and $\operatorname{cov}\left(e_{1}, e_{2}\right)$, with the genetic correlation $\hat{r}=\frac{\operatorname{cov}\left(a_{1}, a_{2}\right)}{\sqrt{\sigma_{a_{1}}^{2}} \sqrt{\sigma_{a 2}^{2}}}$.

\section{Sampling error of the estimated parameters}

From the sampling error of involved (co)variances, we obtain the results of Robertson [25]:

$$
\operatorname{var}\left(\hat{h}_{1}^{2}\right)=\frac{\left(4-h_{1}^{2}\right)^{2}\left[4+(n-1) h_{1}^{2}\right]^{2}}{8 n(n-1)(p-1)}
$$

Assuming $h_{1}^{2}=h_{2}^{2}=h^{2}$, a formula much simpler than that of Tallis [32] is finally obtained:

$$
\operatorname{var}(\hat{r})=\frac{1}{p-1}\left[\left(\frac{\lambda}{n}+1-r^{2}\right)^{2}+\frac{(r \lambda)^{2}}{n(n-1)}\right] .
$$

\section{APPENDIX B: ESTIMATION OF GENETIC PARAMETERS BY DIVERGENT SELECTION OF TWO TRAITS IN TWO GROUPS OF ANIMALS}

\section{Experimental design}

Two traits were measured in two groups of $4 m$ males in generation 1 (G1). Within each group, $n$ extreme animals i.e. a proportion $p$, was selected among the $4 m$. Each of these four groups of $n$ males was mated with $m$ randomly chosen dams, producing $m$ sons (or daughters) in generation 2 (G2), as shown in Figure 1.

\section{Estimated parameters}

In $\mathrm{G} 1$, a selection differential of $2 S_{1}$ for the trait $X_{1}$, and $2 S_{2}$ for $X_{2}$ respectively, is generated between the two groups of $n$ sires, with: $S=z / p, z$ being 
the ordinate of the reduced normal distribution at the X-coordinate truncation corresponding to the $p$ selected animals.

In $\mathrm{G} 2$, the differences between the two groups of offspring in response to the divergent selection of sires are notated:

$R_{1}$ for the direct response for offspring in $X_{1}$ after selection on $X_{1}$, respectively $R_{2}$ for $X_{2}$,

$C_{2}$, for the correlated response in $X_{2}$ after selection on $X_{1}$, respectively $C_{1}$ for $X_{1}$ after selection on $X_{2}$.

If $h_{1}^{2}$ and $h_{2}^{2}$ are the heritability values of $X_{1}$ and $X_{2}$, and $r$ the genetic correlation, the expected phenotypic differences of offspring are:

$$
\begin{gathered}
R_{1}=h_{1}^{2} S_{1}, \\
C_{2}=r h_{1} h_{2} S_{1} .
\end{gathered}
$$

The estimated values of genetic parameters are:

$$
\begin{gathered}
\hat{h}_{1}^{2}=\frac{R_{1}}{S_{1}} \\
\hat{r}=\sqrt{\frac{C_{2}}{R_{1}} \frac{C_{1}}{R_{2}}} .
\end{gathered}
$$

\section{Sampling errors of the estimated parameters}

From the sampling errors of the $R_{i}$ and $C_{i}$ we can obtain:

$$
\begin{gathered}
\operatorname{var}\left(\hat{h}_{1}^{2}\right)=\frac{h_{1}^{2}}{2 S_{1}^{2}}\left[\frac{\left(1-h_{1}^{2}\right)}{n}+\frac{\lambda_{1}}{m}\right] \\
\text { with } \lambda_{1}=\frac{4}{h_{1}^{2}}-1
\end{gathered}
$$

and:

$$
\begin{aligned}
\operatorname{var}(\hat{r})=\frac{1}{2}\left\{\left(\frac{1}{2 S_{2} h_{2}}\right)^{2}\left[\frac{1-r^{2}}{n}+\frac{\lambda_{1}+r^{2} \lambda_{2}}{m}\right]\right. & \\
& \left.+\left(\frac{1}{2 S_{1} h_{1}}\right)^{2}\left[\frac{1-r^{2}}{n}+\frac{\lambda_{2}+r^{2} \lambda_{1}}{m}\right]\right\} .
\end{aligned}
$$

If $S_{1}=S_{2}=S$, and $h_{1}=h_{2}=h$, and $\lambda_{1}=\lambda_{2}=\lambda$, then

$$
\operatorname{var}(\hat{r})=\left(\frac{1}{2 S h}\right)^{2}\left[\frac{1-r^{2}}{n}+\frac{\lambda\left(1+r^{2}\right)}{m}\right] .
$$




\section{Combining divergent selection and half-sib correlations}

Figure 4 shows that the G1 animals are the progeny of randomly chosen $21 \mathrm{G} 0$ sires in the original population. So, the first generation, G1, of the animals involved in the divergent selection scheme are also known as sire halfsibs. After computing the optimal and sub-optimal designs for the two designs, results $\hat{x}_{d i v}$ and $\hat{x}_{h s i b}$ are combined, assuming their independence, by:

$$
\frac{1}{\operatorname{var}(\hat{x})}=\frac{1}{\operatorname{var}\left(\hat{x}_{d i v}\right)}+\frac{1}{\operatorname{var}\left(\hat{x}_{h s i b}\right)} .
$$

To access this journal online: www.edpsciences.org 